\title{
DEFORMED ENERGY LEVELS OF A PSEUDOHARMONIC POTENTIAL: NONRELATIVISTIC QUANTUM MECHANICS
}

\author{
Abdelmadjid Maireche \\ Physics Department, Sciences Faculty, University of M'sila-M'sila-Algeria \\ E-mail:abmaireche@gmail.com
}

\begin{abstract}
The paper described the exact solutions of Schrödinger equation for diatomic molecules with pseudoharmonic potential $V(r)=D\left(\frac{r}{r_{e}}-\frac{r_{e}}{r}\right)^{2}$, it was solved by applying the Boopp's shift method to first order in the non-commutativity parameter $\theta$ and standard perturbation theory, the bound state energy (eigen values) found to be degenerate. It's shown that the noncommutative Hamiltonian (NC) described automatically a particle with spin (1/2) interacted with an external magnetic field, including the usual Zeeman effect in commutative space. Furthermore, I observed the NC Hamiltonian can be described automatically the spin-orbit interaction, I justified my results by calculate the absolute incertitude.
\end{abstract}

Keywords: Star product, Boopp's shift method, diatomic molecules, pseudoharmonic potential.

\section{INTRODUCTION}

Recently, the three fundamental equations: Schrödinger, Klein-Gordon and Dirac were satisfied a beautiful Physics success at low and high energy, respectively. Furthermore gauge theory and standard model was the second revolution in modern Physics after general relativity. The Schrödinger equation played a crucial for describing many Physics phenomena, the exact solutions for a Colombian and for a harmonic oscillator as well as in an arbitrary number of spatial dimensions represents typical models in Quantum mechanics [1-18]. Although a new concepts of space-time, known by of noncommutative spaces, represent a hop to obtained a new and profound interpretations at microscopic scales, the noncommutativity is introduced by many ways, the simple approach, it consider the position and momentum operators obeys to the Heisenberg commutation relation, that is similarly to quantize space-time coordinates, when the commutators $\left\lfloor\hat{x}_{i}, \hat{x}_{j}\right\rfloor \neq 0$ [22], the formalism of star product, Boopp's shift method and the Seiberg-Witten map are plays a fundamental roles in this new theory. The rich mathematical structure of the noncommutative theory gives a rise to the hop to get a better understanding of Physics phenomena at smallness distances and hop to solve above mentioned problems. The Physics idea of a noncommutative space satisfied by on based, in addition, to usual Quantum commutators, the following special commutators [18-36]: 


$$
\left[\hat{x}_{i}^{*}, \hat{x}_{j}\right]=i \theta_{i j}
$$

The parameters $\theta^{i j}$ are an antisymmetric real matrix of dimension square length in the noncommutative canonical-type space, throughout this paper the natural unites $(c=\hbar=1)$ and $(\vec{\mu}=\dot{s})$ are employed. It's important to notice that, the fundamental commutator which represented by eq. (1), it has been deduced from the star product between two arbitrary functions $f(x)$ and $g(x)$ in the first order of $\theta[19,20,35,36]$ :

$$
f(x) * g(x)=f(x) g(x)-\frac{i}{2} \theta^{\alpha \beta} \partial_{\alpha} f(x) \partial_{\beta} g(x)
$$

Thus, the usual product $f(x) g(x)$ replace by new star product $f(x) * g(x)$ which represented by eq (2). Actually there are many attempts to study noncommutative space time in three dimensional spaces, but limited Physics phenomenons are studied in two dimensional spaces for examples (the isotropic Harmonic oscillator plus inverse quadratic, the sextic, the singular even-power, the mixed, the Inverse-Power) the study of bound-states of the Schrödinger equation for the previously potentials is fundamental to understanding the molecular spectrum of a diatomic molecule, as a good example, of a Physics interaction, in two dimensional space (2D), the pseudoharmonic potential play an important role in the history of molecular and Quantum Chemistry, furthermore, this potential used to describing molecular structures and interactions, in addition, the pseudoharmonic potential may be used for the energy spectrum of linear and non-linear systems [37]. The plan of the paper is as follows: In next section, we present and review the Boopp's shift method. In section 3 a two dimensional central potential known by pseudoharmonic potential is represented. Next, in section 4 we derive the deformed molecular diatomic with the pseudoharmonic potential in noncommutative real space, we also obtain the deformed bound-state eigensolutions of molecular diatomic. A detailed discussion presented in section 5 . Finally, the relevant conclusions are given in last section.

\section{REVIEW OF BOOPP'S SHIFT METHOD}

In this paper, a Boopp's shift method will be used, instead of solving the non commutative Schrödinger equation by using star product procedure, we replace new star product in the Schrödinger equation by the usual ordinary product by making a Boopp's shift and the translation of the noncommutative Schrödinger equation, respectively, $[26,35,36]$ :

$$
\begin{aligned}
& \hat{x}_{i}=x_{i}-\frac{\theta_{i j}}{2} p \text { and } \quad \hat{p}_{i}=p_{i} \\
& \hat{H}\left(\hat{p}_{i}, \hat{x}_{i}\right) * \Psi(\overrightarrow{\tilde{r}})=E_{n c-p h} \Psi\left(\frac{\vec{r}}{\vec{r}}\right) \rightarrow H\left(\hat{p}_{i}, \hat{x}_{i}\right) \psi(\vec{r})=E_{n c-p h} \psi(\vec{r})
\end{aligned}
$$

where $\hat{H}\left(\hat{p}_{i}, \hat{x}_{i}\right), \hat{\Psi}(\ddot{\vec{r}})$ and $E_{n c-p h}, \psi(\vec{r})$ are represents NC 2D-Hamiltonian, NC complex wave function, NC energy and ordinary complex wave function respectively. After a straightforward calculation, on can show that in NC 2D spaces:

$$
\begin{aligned}
& \hat{x}=x-\frac{\theta}{2} p_{y}, \quad \hat{\mathrm{y}}=y+\frac{\theta}{2} p_{x} \\
& \hat{x}^{2}=x^{2}-\theta x p_{y}, \quad \hat{y}^{2}=y^{2}+\theta y p_{x} \\
& \hat{p}_{x, y}=p_{x, y}
\end{aligned}
$$

where $\theta \equiv \theta^{12}$ then, we can easily verify that, the commutators, between the operators position themselves, in NC $2 \mathrm{D}$ space, will be written as follows:

$$
\begin{gathered}
{[\hat{x}, \hat{y}]=i \theta} \\
{[\hat{x}, \hat{x}]=[\hat{y}, \hat{y}]=0} \\
{[x, y]=[x, x]=[y, y]=0}
\end{gathered}
$$


Based to the eq. (4), we can show after a straightforward calculation, the following two-terms, which allow us to obtain the deformed potential in 4th section:

$$
\begin{aligned}
& \hat{r}^{2}=r^{2}-\theta L_{z} \\
& \frac{1}{\hat{r}^{2}}=\frac{1}{r^{2}}+\frac{\theta}{r^{4}} L_{z}
\end{aligned}
$$

As a direct result of eq. (4), it's clearly that, the kinetic term is invariant in NC 2D spaces:

$$
\frac{\vec{p}^{2}}{2 \mu}=\frac{\vec{p}^{2}}{2 \mu}
$$

where ${ }^{\mu}$ is the reduced mass of the diatomic molecules.

\section{THE PSEUDOHARMONIC POTENTIAL IN ORDINARY TWO DIMENSIONAL SPACES}

In this section we shall review some fundamentals production of Schrödinger equation with pseudoharmonic potential while referring to [37] for more details. In particular, we shall have for previously central potential in commutative $2 \mathrm{D}$ space:

$$
\left(-\frac{1}{2 \mu}\left(r \frac{1}{r} \frac{\partial}{\partial r}\left(r \frac{\partial}{\partial r}\right)+\frac{1}{r^{2}} \frac{\partial^{2}}{\partial \varphi^{2}}\right)+V(r)\right) \Psi(\dot{r})=E \Psi(\dot{*}),
$$

with, pseudoharmonic potential:

$$
V(r)=D_{e}\left(\frac{r}{r_{e}}-\frac{r_{e}}{r}\right)^{2}
$$

where $D_{e}$, is the dissociation energy and $r_{e}$ is the equilibrium intermolecular separation. The potential (9) can be rewritten in the form of an isotropic Harmonic oscillator plus inverse quadratic potential [37]:

$$
V(r)=a r^{2}+\frac{b}{r^{2}}+c
$$

where $a=D_{e} r_{e}^{-2}, b=D_{e} r_{e}^{+2}$ and ${ }^{c=-2 D_{e}}$. The complete normalized wave function $\Psi(\vec{r})$ separated on radial function $R_{n l}(r)$ and a standard angular as follows:

$$
\Psi(\dot{r})=r^{-\frac{1}{2}} R_{m}(r) \exp ( \pm i m \varphi)
$$

where $R_{n l}(r)$ satisfy the following equation:

$$
\left(\frac{d^{2}}{d r^{2}}+2 \mu\left(E-a r^{2}-\frac{b}{r^{2}}-c\right)-\frac{m^{2}-\frac{1}{4}}{r^{2}}\right) R_{m}(r)=0
$$

where $E$ is the energy and $m$ represent the orbital angular momentum Quantum number. The complete orthonormalization functions and the energy eigen values respectively in two dimensional spaces [37]:

$$
\begin{gathered}
\Psi_{0}(r, \varphi)=N_{0} \exp \left(-\frac{1}{2} \sqrt{2 a \mu} r^{2}\right) r^{\sqrt{2 b \mu}} \\
E_{0}=c+\sqrt{\frac{2 a}{\mu}}(1+\sqrt{2 b \mu}) \\
\Psi_{2}(r, \varphi)=N_{2} \exp \left(-\frac{1}{2} \sqrt{2 a \mu} r^{2}\right) r \sqrt{2 b \mu+4} \exp ( \pm i 2 \varphi) \\
E_{1}=c+\sqrt{\frac{2 a}{\mu}}(1+\sqrt{2 b \mu+1}) \\
\Psi_{2}(r, \varphi)=N_{2} \exp \left(-\frac{1}{2} \sqrt{2 a \mu} r^{2}\right) r \sqrt{2 b \mu+4} \exp ( \pm i 2 \varphi) \\
E_{2}=c+\sqrt{\frac{2 a}{\mu}}(1+\sqrt{2 b \mu+4}) \\
\Psi_{m}(r, \varphi)=N_{m} \exp \left(-\frac{1}{2} \sqrt{2 a \mu} r^{2}\right) r \sqrt{2 b \mu+m^{2}} \exp ( \pm i m \varphi) \\
E_{m}=c+\sqrt{\frac{2 a}{\mu}}\left(1+\sqrt{2 b \mu+m^{2}}\right),
\end{gathered}
$$

where, the normalization constant ${ }^{N_{m}}$ is given by:

$$
N_{m}=\left[\frac{(2 \sqrt{2 a \mu})^{1+\sqrt{2 b \mu+m^{2}}}}{\sqrt{2 b \mu+m^{2}} !}\right]^{\frac{1}{2}}
$$

As well know that, pseudoharmonic potential is a modified Harmonic oscillator were studied. 


\section{THE PSEUDOHARMONIC POTENTIAL IN NONCOMMUTATIVE TWO DIMENSIONAL REAL SPACES}

If we apply the Boopp's shift method to the Schrödinger equation, on the molecular diatomic with the pseudoharmonic potential $V(\hat{r})$, we obtain:

$$
\left(-\frac{1}{2 \mu}\left(r \frac{1}{r} \frac{\partial}{\partial r}\left(r \frac{\partial}{\partial r}\right)+\frac{1}{r^{2}} \frac{\partial^{2}}{\partial \varphi^{2}}\right)+V(\hat{r})\right) \hat{\Psi}(\dot{r})=E_{N C} \hat{\Psi}(\dot{r})
$$

where $V(\hat{r})$ obtained from replaced operator $\hat{r}$ by the ordinary $r$ as follows:

$$
V(\hat{r})=a \hat{r}^{2}+b \hat{r}^{-2}+c
$$

And $E_{N C}$ is the energy in NC 2D space. Based on eq. (6) we can show that, the operator potential $V(\hat{r})$ take the following form:

$$
V(\hat{r})=a r^{2}+\frac{b}{r^{2}}+c+V_{p h}(r)
$$

where the supplementary term $V_{p h}(r)$ take the form:

$$
V_{p h}(r)=\theta\left(\frac{b}{r^{4}}-a\right) L_{z}
$$

Clearly, the term ${ }^{V_{\text {pot }}}(r)$, proportional with small parameter $\theta$, then we considered as a perturbative terms, furthermore we can rewritten it to the equivalent form:

$$
V_{p h}(r)=2 \theta f(r) \overleftrightarrow{S} \overleftrightarrow{L}
$$

where $\vec{S}=\frac{\overrightarrow{1}}{2}$ and $f(r)=\frac{b}{r^{4}-a}$. The operator $\overleftrightarrow{S} \vec{L}$ traduced physically, the coupling between spin and orbital momentum, known by spinorbit interaction. Furthermore we can rewrite it to the equivalent form:

$$
V_{p h}(r)=\theta f(r)\left(\vec{J}^{2}-\vec{L}^{2}-\overleftrightarrow{S}^{2}\right)
$$

The corresponding NC Hamiltonian $H_{N C-1}$ will be form, as follows:

$$
H_{N C-1}=-\frac{\Delta}{2 \mu}+D_{e}\left(\frac{r}{r_{e}}-\frac{r_{e}}{r}\right)^{2}+\theta f(r)\left(\vec{J}^{2}-\vec{L}^{2}-\vec{S}^{2}\right)
$$

It known that, in Quantum mechanics the operators $\left(\vec{J}^{2}, \overleftrightarrow{L}^{2}, \overleftrightarrow{S}^{2}\right.$ and $\left.J_{z}\right)$ are formed complete set of commuting observables, then $\left(\vec{J}^{2}-\vec{L}^{2}-\vec{S}^{2}\right)$ will be gives two eigen values: the first is $\left(L(j, l, s)=\left(l+\frac{1}{2}\right)\left(l+\frac{3}{2}\right)-l(l+1)-\frac{3}{4}\right)$ and

the second $\left(L^{\prime}(j, l, s)=\left(l-\frac{1}{2}\right)\left(l+\frac{1}{2}\right)-l(l+1)-\frac{3}{4}\right)$, corresponded $^{j=l+\frac{1}{2}}$ (spin up) and ${ }^{j=l-\frac{1}{2}}$ (spin down), respectively. Then, we can form a diagonal matrix of order $(2 \times 2)$ with elements $H_{u p}$ and ${ }^{H_{d}}$ :

$$
\begin{gathered}
H_{u p}=-\frac{\Delta}{2 \mu}+D_{e}\left(\frac{r}{r_{e}}-\frac{r_{e}}{r}\right)+\theta f(r) L(j, l, s) \text { if } j=l+\frac{1}{2} \Rightarrow \text { spinup } \\
\left.H_{d}=-\frac{\Delta}{2 \mu}+D_{e}\left(\frac{r}{r_{e}}-\frac{r_{e}}{r}\right)+\theta f(r)\right) L^{\prime}(j, l, s) \text { if } j=l-\frac{1}{2} \Rightarrow \text { spindown }
\end{gathered}
$$

The modification to the energy levels, associate with spin up and spin down, $E_{u p}$ and $E_{d}$, respectively, at first order of $\theta$, obtains by the applying the perturbation theory:

$$
\begin{aligned}
& E_{u p}=\left\langle n, j, l, s\left|H_{u p}\right| n, j, l, s\right\rangle \\
& E_{d}=\left\langle n, j, l, s\left|H_{d}\right| n, j, l, s\right\rangle
\end{aligned}
$$

In NC 2D real space, the modification of the energy levels associated with spin up, in the first order of $\theta$ correspond the $m^{\text {th }}$ order of excited states $E_{m}$ determined by using Eqs. (19), (20) and (23):

$$
\frac{E_{m u p}}{\left|N_{m}\right|^{2}}=\theta L(j, l, s) \int_{0}^{+\infty}\left[\exp \left(-\frac{1}{2} \sqrt{2 a \mu} r^{2}\right) r^{\sqrt{2 b \mu+m^{2}}}\right]^{2}\left(\frac{b}{r^{4}}-a\right) r d r
$$


this can be written as follows:

$E_{1 m u p}=2 \pi \theta L(j, l, s) \frac{(2 \sqrt{2 a \mu})^{1+\sqrt{2 b \mu+m^{2}}}}{\sqrt{2 b \mu+m^{2}} !} \theta\left(b T_{1}-a T_{2}\right)$

where the two terms $T_{1}$ and $T_{2}$ are given by:

$$
\begin{aligned}
& T_{1}=\int_{0}^{+\infty} \exp \left(-\sqrt{2 a \mu} r^{2}\right) r^{2 \sqrt{2 b \mu+m^{2}}-3} d r \\
& T_{2}=\int_{0}^{+\infty} \exp \left(-\sqrt{2 a \mu} r^{2}\right) r^{1+2 \sqrt{2 b \mu+m^{2}}} d r
\end{aligned}
$$

and we use the following form of special integral [39]:

$$
\int_{0}^{+\infty} x^{v-1} \exp \left(-\beta x^{2}-\gamma x\right) d x=(2 \beta)^{-\frac{v}{2}} \Gamma(v) \exp \left(\frac{\gamma^{2}}{8 \beta}\right) D_{-v}\left(\frac{\gamma}{\sqrt{2 \beta}}\right)
$$

where $D_{-v}$, is the parabolic cylinder function, as a condition $(\operatorname{Re} l(\beta) \succ 0$ and $\operatorname{Re} l(v) \succ 0)$, we set $\beta=\sqrt{2 a \mu}, \quad \gamma=0$ and $\left(v=2 \sqrt{2 b \mu+m^{2}}-2\right.$ in $T_{1}$ and $v=2+2 \sqrt{2 b \mu+m^{2}}$ in $^{T_{2}}$ ), which allow us to obtaining:

$$
\begin{gathered}
T_{1}=(2 \sqrt{2 a \mu})-\left(\sqrt{2 b \mu+m^{2}}-1\right) \Gamma\left(2\left(\sqrt{2 b \mu+m^{2}}-1\right)\right) D_{-2\left(\sqrt{2 b \mu+m^{2}}-1\right)}(1) \\
T_{2}=(2 \sqrt{2 a \mu})^{-\left(\sqrt{2 b \mu+m^{2}}+1\right)} \Gamma\left(2\left(\sqrt{2 b \mu+m^{2}}+1\right)\right) D_{-2\left(\sqrt{2 b \mu+m^{2}}+1\right)}(1)
\end{gathered}
$$

the above equations are simplified to the following form:

$$
E_{u p}=2 \pi L(j, l, s)\left|N_{m}\right|^{2} \theta \delta,
$$

Where ${ }^{\alpha}, \beta$ and $\delta$ are given by:

$$
\begin{aligned}
& \alpha=\sqrt{2 b \mu+m^{2}}-1, \beta=\left(\sqrt{2 b \mu+m^{2}}+1\right) \\
& \delta=\left(b(2 \sqrt{2 a \mu})^{-\alpha} \Gamma(2 \alpha) D_{-2 \alpha}(1)-a(2 \sqrt{2 a \mu})^{-\beta} \Gamma(2 \beta) D_{-2 \beta}(1)\right)\left|N_{m}\right|^{2}
\end{aligned}
$$

Similarly, we can easily obtain the modification the energy levels, corresponding electron with spin down $E_{m d}$ in the first order of $\theta$, corresponding $\mathrm{m}^{\text {th }}$ order of excited states, as follows:

$$
E_{m_{d}}=2 \pi L^{\prime}(j, l, s)\left|N_{m}\right|^{2} \theta \delta
$$

We conclude, from Eqs. (13), (29) and (31) that, the total energy of an electron with spin up and spin down ( $\left.\begin{array}{lll}E_{N C m_{u p}} & E_{N C m_{d}}\end{array}\right)$ corresponded ( ${ }^{H_{u p}}$ and ${ }^{H_{d}}$ ) are given by, respectively:

$$
\begin{aligned}
& E_{N C m_{u p}}=c+\sqrt{\frac{2 a}{\mu}}\left(1+\sqrt{2 b \mu+m^{2}}\right)+2 \pi L(j, l, s)\left|N_{m}\right|^{2} \theta \delta \\
& E_{N C m_{d}}=c+\sqrt{\frac{2 a}{\mu}}\left(1+\sqrt{2 b \mu+m^{2}}\right)+2 \pi L^{\prime}(j, l, s)\left|N_{m}\right|^{2} \theta \delta
\end{aligned}
$$

\section{DISCUSSIONS}

As a good example, of applications for obtained results, we can estimate the emission energy $\Delta E_{N C d}$, corresponding electron with spin down, when translate from first excited states to the stationary state, as follows:

$$
\Delta E_{N C d}=E_{N C l d}-E_{N C O d}
$$

Using eq. (32), we obtain easily:

$$
\frac{\Delta E_{N C d}-\Delta E}{2 \pi \delta \theta}=L^{\prime}\left(\frac{3}{2}, 1, \frac{1}{2}\right)\left|N_{1}\right|^{2}-L^{\prime}\left(\frac{3}{2}, 0, \frac{1}{2}\right)\left|N_{0}\right|^{2}
$$

where represent the emission energy in the ordinary commutative space:

$$
\Delta E=\sqrt{\frac{2 a}{\mu}}\left(\sqrt{2 b \mu+1^{2}}-\sqrt{2 b \mu}\right)
$$

The two values $L^{\prime}\left(\frac{3}{2}, 1, \frac{1}{2}\right)=-2$ and $L^{\prime}\left(\frac{3}{2}, 0, \frac{1}{2}\right)=-1$, then, the eq. (34) reduce to the simple form:

$$
\Delta E_{N C d}=\Delta E+2 \pi\left(\left|N_{0}\right|^{2}-2\left|N_{1}\right|^{2}\right) \theta \delta
$$


thus, the noncommutativity modified $\Delta E_{u p}$ by $2 \pi\left(-2 \frac{(2 \sqrt{2 a \mu})^{1+\sqrt{2 b \mu+1}}}{\sqrt{2 b \mu+1}}+\frac{(2 \sqrt{2 a \mu})^{1+\sqrt{2 b \mu}}}{\sqrt{2 b \mu}}\right) \theta \delta$ it's important to calculate the absolute incertitude:

$$
\frac{\left|\Delta E_{N C d}-\Delta E\right|}{\Delta E_{d}}=\frac{2 \pi\left(\frac{(2 \sqrt{2 a \mu})^{1+\sqrt{2 b \mu}}}{\sqrt{2 b \mu}}-2 \frac{(2 \sqrt{2 a \mu})^{1+\sqrt{2 b \mu+1}}}{\sqrt{2 b \mu+1}}\right) \theta \delta}{\sqrt{\frac{2 a}{\mu}}\left(\sqrt{2 b \mu+1^{2}}-\sqrt{2 b \mu}\right)+2 \pi\left(\left|N_{0}\right|^{2}-2\left|N_{1}\right|^{2}\right) \theta \delta}
$$

after a straightforward calculation, we get the absolute incertitude:

$$
\frac{\left|\Delta E_{N C d}-\Delta E\right|}{\Delta E_{d}}=2 \pi\left(\frac{(2 \sqrt{2 a \mu})^{1+\sqrt{2 b \mu}}}{\sqrt{2 b \mu}}-2 \frac{(2 \sqrt{2 a \mu})^{1+\sqrt{2 b \mu+1}}}{\sqrt{2 b \mu+1}}\right) \theta \delta \prec \measuredangle E
$$

we observe that the obtained result $\left(2 \pi\left(\frac{(2 \sqrt{2 a \mu})^{1+\sqrt{2 b \mu}}}{\sqrt{2 b \mu}}-2 \frac{(2 \sqrt{2 a \mu})^{1+\sqrt{2 b \mu+1}}}{\sqrt{2 b \mu+1}}\right) \theta \delta\right)$

proportional to the infinitesimal parameter, then, the absolute incertitude is justified. Regarding to the eq. (32), the eigen values of energies are real, and then the $\mathrm{NC}$ Hamiltonian is Hermitian $\left(H_{N C-1}=\left(H_{N C-1}\right)^{+}\right)$, which can be decomposed as follows:

$$
H_{N C-1}=H_{p h-c o m}+H_{p h-s o}
$$

where ${ }^{H_{p h-c o m}}$ and ${ }^{H_{p h-s o}}$ are determined from, the following relation, respectively:

$$
\begin{aligned}
H_{p h-c o m} & =\left[-\frac{\Delta}{2 \mu}+D_{e}\left(\frac{r}{r_{e}}-\frac{r_{e}}{r}\right)^{2}\right] I_{2 * 2} \\
H_{p h-s o} & =\theta f(r)\left(\begin{array}{cc}
L(j, l, s) & 0 \\
0 & L^{\prime}(j, l, s)
\end{array}\right)
\end{aligned}
$$

It's clearly ${ }^{H_{p h-c o m}}$ represent a particle of spin $(1 / 2)$ interacted exactly with the pseudoharmonic potential in ordinary $2 \mathrm{D}$ space, while the matrix ${ }^{H_{p h-s o}}$ represent the spin-orbit interaction.
Furthermore, if we choose, the parameter $\theta$ and the vector of a magnetic field, which oriented with $(\mathrm{Oz}$.$) axes, as follows [35,36]$ :

$$
\begin{aligned}
\theta & =\alpha B, \stackrel{\leftrightarrow}{B}=B \dot{k} \\
\theta L_{z} & =\alpha\left(\stackrel{\leftrightarrow}{J} \vec{B}-H_{Z}\right)
\end{aligned}
$$

where $\alpha$ is real proportional constant and $H_{Z}$ is the usual Zeeman operator in commutative space, Substituting Eq. (41) into the NC Hamiltonian equation (40), we find:

$$
H_{N C-2}=-\frac{\Delta}{2 \mu}+D_{e}\left(\frac{r}{r_{e}}-\frac{r_{e}}{r}\right)^{2}+H_{p h-m a g}
$$

where the obtained magnetic operator $H_{p h-m a g}$ is given by:

$$
H_{p h-m a g}=-\alpha f(r)\left(H_{Z}-\overleftrightarrow{J} \overleftrightarrow{B}\right)
$$

Physically, the eq. (43) represents the new magnetic interactions between electron with spin $(1 / 2)$ and an external magnetic field, including the ordinary Zeeman effect. In another hand, it's evidently to consider the infinitesimal parameter $\theta$, the sum of 2infinitesimal parameters $\theta_{1}$ and $\theta_{2}$, then the complete NC Hamiltonian equal the sum of three fundamentals parts, the first one and the second part, are was represented from eq. (40), while the last part, it has seen from the eq. (43). Thus, the final operator of $\mathrm{NC}$ Hamiltonian ${ }^{H_{P h-n c}}$, which obtain by applying the superposition principal:

$$
H_{P h-n c}=H_{p h-c o m}+H_{p h-s o}+H_{p h-m a g}
$$

where, the 3-operators $H_{p h-c o m}, H_{p h-c o m}$ and $H_{p h-m a g}$ are given by: 


$$
\begin{aligned}
& H_{p h-c o m}=-\frac{\Delta}{2 \mu}+D_{e}\left(\frac{r}{r_{e}}-\frac{r_{e}}{r}\right)^{2} \\
& H_{p h-s o}=\alpha\left(\frac{b}{r^{4}}-a\right)\left(\overleftrightarrow{J}^{2}-\overleftrightarrow{L}^{2}-\overleftrightarrow{S}^{2}\right) \\
& H_{p h-m a g}=\theta\left(\frac{b}{r^{4}}-a\right)\left(\overleftrightarrow{J} \overleftrightarrow{B}-H_{Z}\right)
\end{aligned}
$$

Regarding the eq. (33), we can deduce the complete NC Quantum spectrum of diatomic molecules with pseudoharmonic potential: $E_{N C u p}(j, l, s, m)$ and $E_{N C d}(j, l, s, m)$ corresponding spin up and spin down respectively, evidently equal the sum 3 values of energies: $\left(E_{m}, E_{N C-s o}, E_{N C-m}\right)$ corresponding $\left(H_{p h-c o m}, H_{p h-s o}\right.$ and $\left.H_{p h-m a g}\right)$, respectively, as follows:

$$
\begin{aligned}
& E_{N C u p}(j, l, s, m)=c+\sqrt{\frac{2 a}{\mu}}\left(1+\sqrt{2 b \mu+m^{2}}\right)+ \\
& +2 \pi L \frac{(2 \sqrt{2 a \mu})^{1+\sqrt{2 b \mu+m^{2}}}}{\sqrt{2 b \mu+m^{2}} !} \delta\left(L(j, l, s) \theta_{1}+m \theta_{2}\right) \\
& E_{N C d}(j, l, s, m)=c+\sqrt{\frac{2 a}{\mu}}\left(1+\sqrt{2 b \mu+m^{2}}\right)+ \\
& +2 \pi L \frac{(2 \sqrt{2 a \mu})^{1+\sqrt{2 b \mu+m^{2}}}}{\sqrt{2 b \mu+m^{2}} !} \delta\left(L^{\prime}(j, l, s) \theta_{1}+m \theta_{2}\right)
\end{aligned}
$$

\section{CONCLUSIONS}

In this paper, the Schrödinger equation for pseudoharmonic potential was solved by applying the Boopp's shift method to first order of the parameter in non commutative two dimensional spaces and ordinary stationer perturbation theory, we derived two new parts of NC Hamiltonian operator, the full modified of the energies levels corresponding electron with spin up and spin down were established. From this recent work, we concluded that, the NC Hamiltonian interpreting automatically two fundamentals Physics phenomenon: the new Zeeman magnetic effect including ordinary Zeeman effect and the spin-orbit interaction. Thus, the noncommutativity properties for pseudoharmonic potential were prolonged to include new symmetries can be considers as a good candidate to gives new applications in another Physics fields.

\section{ACKNOWLEDGEMENTS:}

This work was supported by research laboratory of: Physique et Chimie des matériaux, in university of M'sila, Algeria.

\section{REFERENCES}

[1] Dong, S.H. Int J Theor Phys. (2001) 40, 2, 559567.

[2] Liboff, R. L. Introductory Quantum mechanics, 4th ed., Addison Wesley: San Francisco, CA, 2003.

[3] Dong, S.H.; Lozada-Cassou, M. American Journal of Applied Sciences. (2005) 2, 1, 376382.

[4] Elbaz, E. Quantum. Springer: Verlag Berlin Heidelberg, New York, 1998.

[5] Chang, T. and Fong, L. Li. Gauge theory of elementary particle Physics, Clarendon PressOxford: USA, 1988.

[6] Vanden Berghe, G.; De Meyer, H. and Van Daele, M. Int J Theor Phys. (1995) 34, 9, 1931 1937.

[7] Landau, L. D.; Lifshitz, E. M. Quantum Mechanics Relativistic Theory, 3rd ed., Cambridge University Press: London, 1997.

[8] Dong, S.H.; Guo-Hua, S. Foundations of Physics Letters. (2003) 16，4, 357-366

[9] Dong, S.H. Int J Theor Phys. (2000) 39, 4, 1119-1128.

[10] Tapas, D; Subhankar, R. and Altug, A. Exact Solution of N-

Schrödinger Equation with Pseudoharmonic Potential via Laplace Transforme Approach, arXiv: 1308.5295v1 [math-ph] 24 Aug 2013.

[11] Hassoun, G. Q. Am. J. Phys. (1981) 49, 2, 143146.

[12] Dong, S.H. Foundations of Physics Letters. (2002) 15, 4, 385-395.

[13] Khan, G. R. Eur. Phys. J. (2009) 53, 123-125.

[14] Buragohain, L. and Ahmed, S. A. S. Lat. Am. J. Educ.(2009) 3, 573.

[15] Buragohain, L. and Ahmed, S. A. S. Lat. Am. J. Phys. Educ. (2010) 4, 1, 79-83. 
[16] Nabaratna, B. Cent. Eur. J. Phys. 12, 4, 256-265 (2014).

[17] Sameer, M. Ikhdair and Ramazan Sever. Cent.Eur. J. Phys. (2008) 6, 3, 685-696.

[18] Anjana, Sinha. Int J Theor Phys. (1998) 37, 7, 2055- 2065.

[19] L. Mezincescu, UMTG-233, Star product in Quantum Mechanics, arXiv: hep th/0007046v2 21 Jul. 2000.

[20] Yuan, Yi; LI Kang; WANG Jain-Hua; CHEN Chi-Yi. Chinese Physics C. (2010) 34, 5,543547.

[21] Mirza B.; Mohadesi M. Commun. Theor. Phys. 42, 664 (2004).

[22] Snyder, H. S. Phys. Rev, (1947) 71, 38.

[23] Manin, Y. I. Math. Phys. (1989) 123, 163.

[24] Seiberg, N.; Witten E. JHEP. (1999) 09:032.

[25] J. Madore, S.; Schraml, P.; Schupp, and J. Wess. Gauge theory on noncommutative space, Eur. Phys. J. C16, 161 (2000), hep-th/0001203.

[26] Wang, J. H.; Li, K.; Dulat, S. Chinese Physics C. (2008) 32, 10, 803-806.

[27] Mirza, B.; Zarei, M. Eur. Phys. J. C. (2004) 32, 583.
[28] Belluchi, S. Phys. Lett, B. (2001) 552, 345.

[29] Acatrinei, C. JHEP. (2001) 0109.

[30] Chaichian, M.; Sheikh-Jabbari, M. M. and Sheikh-Jabbari, A. Tureanu, Phys. Rev. Lett. (2001) 86, 2716.

[31] Connes, A.; Douglas, M. R. and Schwarz, A. JHEP. 9802, 003; hep- th/9808042 (1998).

[32] Douglas, M. R. and Nekrasov, N. A. Rev. Mod. Phys. 73, 977; hep-th/0106048 (2001).

[33] Sag, M. and Goodisman, J. Am. J. Phys. (1985) 53,350-355.

[34] Le Roy, R. J. and Bernstein, R. B. J. Chem. Phys. (1970) 52, 3869-3879.

[35] Maireche, A. African review of Physics. (2014) $9,185-195$.

[36] Maireche, A. Life Sciences, (2014) 11, 6.

[37] Sameer, M.; Ramazan Sever. CEJP. (2007) 5, 4, 516-527.

[38] Sameer, M.; Ramazan Sever. CEJP. (2008) 6, 3, 685-696.

[39] Gradshteyn, I. S. and Ryzhik, I. M. Table of Integrals, Series and Products, 7th. ed.; Elsevier, 2007. 
يصف البحث الحلول الدقيقة لمعادلة شرودينغر لجزئ ثنائي الذرة ينأثر بكمون نوافقي كاذب Boopp's shift في المرتبة الأولى للوسيط اللاتبادلي $،$ ، ونظرية $V(r)=D\left(\frac{r}{r_{e}}-\frac{r_{e}}{r}\right)$ الاضطر ابات المستقرة. وقد وجد أن عصابات الطاقة (القيم الذاتية) كانت متحللة. كما وجد أن الهاملتونيان اللاتبادلي يصف ذاتياً جسيم فرميوني يدور نصف دورة ويتفاعل مع مجال مغناطيسي خارجي يتضمن تأثير زيمان المعتاد في الفضاء التبادلي. بالإضافة لذلك لوحظ أن الهاملتونيان اللاتبادلي بإمكانه وصف ظاهرة الدوران في مدار التفاعل تلقائياً و نم تبرير النتائج من خلال حساب الإرتياب المطلق. 